\title{
Modifier -25 and the New 2021 E/M Codes: Documentation of Separate and Distinct Just Got Easier
}

\author{
Howard W. Rogers, MD, PhD
}

\section{PRACTICE POINTS}

- Insurer scrutiny of same-day evaluation and management $(E / M)$ and procedure services has increased, and dermatologists should be prepared for more frequent medical record reviews and audits.

- The new $2021 \mathrm{E} / \mathrm{M}$ codes actually reduce the hurdles for reporting a separate and distinct E/M service by eliminating the history and physical examination bullet points of the previous code set.
The ability to provide same-day evaluation and management (E/M) and procedure services (reported with a modifier -25) is critical to efficient dermatologic care. However, insurer policies that deny or decrease payment modifier -25 and increased frequency of insurer modifier -25 audits are challenging dermatologists' ability to deliver timely, patient-centered care. Understanding the documentation requirements that qualify for billing the minor procedure on the same day as an E/M service is critical for passing medical record reviews. Moreover, the new E/M codes for 2021 have notably decreased documentation burden and have simplified reporting modifier -25 .

Cutis. 2021;108:131-132, 162.

\section{Insurers Target Modifier -25}

Modifier -25 allows reporting of both a minor procedure (ie, one with a 0 - or 10-day global period) and a separate and distinct evaluation and management $(\mathrm{E} / \mathrm{M})$ service on the same date of service. ${ }^{1}$ Because of the multicomplaint nature of dermatology, the ability to report a same-day procedure and an E/M service is critical for efficient, cost-effective, and patient-centered dermatologic care. However, it is well known that the use of modifier -25 has been under notable insurer scrutiny and is a common reason for medical record audits. ${ }^{2,3}$ Some insurers have responded to increased utilization of modifier -25 by cutting reimbursement for claims that include both a procedure and an E/M service or by denying one of the services altogether. ${ }^{4-6}$ The Centers for Medicare and Medicaid Services also have expressed concern about this coding combination with proposed cuts to reimbursement. ${ }^{7}$ Moreover, the Office of Inspector General has announced a work plan to investigate the frequent utilization of E/M codes and minor procedures by dermatologists. ${ }^{8}$ Clearly, modifier -25 is a continued target by insurers and regulators; therefore, dermatologists will want to make sure their coding and documentation meet all requirements and are updated for the new E/M codes for 2021.

The American Medical Association's Current Procedural Terminology indicates that modifier - 25 allows reporting of a"significant, separately identifiable evaluation and management service by the same physician or other qualified health care professional on the same day of a procedure or other service." ${ }^{1}$ Given that dermatology patients typically present with multiple concerns, dermatologists commonly evaluate and treat numerous conditions during one visit. Understanding what constitutes a separately identifiable E/M service is critical to bill accurately and to pass insurer audits.

\section{Global Surgical Package}

To appropriately bill both a procedure and an E/M service, the physician must indicate that the patient's condition required an E/M service above and beyond the usual work of the procedure. The compilation of evaluation and work included in the payment for a procedure is called the global surgical package. ${ }^{9}$ In general, the global surgical package includes local or topical anesthesia; the surgical service/procedure itself; immediate postoperative 
care, including dictating the operative note; meeting/ discussing the patient's procedure with family and other physicians; and writing orders for the patient. For minor procedures (ie, those with either 0- or 10-day global periods), the surgical package also includes same-day E/M services associated with the decision to perform surgery. An appropriate history and physical examination as well as a discussion of the differential diagnosis, treatment options, and risk and benefits of treatment are all included in the payment of a minor procedure itself. Therefore, an evaluation to discuss a patient's condition or change in condition, alternatives to treatment, or next steps after a diagnosis related to a treatment or diagnostic procedure should not be separately reported. Moreover, the fact that the patient is new to the physician is not in itself sufficient to allow reporting of an E/M service with these minor procedures. For major procedures (ie, those with 90-day postoperative periods), the decision for surgery is excluded from the global surgical package.

\section{E/M Codes Simplify Documentation}

The biggest coding change of 2021 was the new E/M codes ${ }^{10}$ Prior to this year, the descriptors of E/M services recognized 7 components to define the levels of E/M services ${ }^{11}$ : history and nature of the presenting problem; physical examination; medical decision-making (MDM); counseling; coordination of care; and time. Furthermore, history, physical examination, and MDM were all broken down into more granular elements that were summed to determine the level for each component; for example, the history of the presenting problem was defined as a chronological description of the development of the patient's present illness, including the following elements: location, quality, severity, duration, timing, context, modifying factors, and associated signs and symptoms. Each of these categories would constitute bullet points to be summed to determine the level of history. Physical examination and MDM bullet points also would be summed to determine a proper coding level. ${ }^{11}$ Understandably, this coding scheme was complicated and burdensome to medical providers.

The redefinition of the E/M codes for 2021 substantially simplified the determination of coding level and documentation. ${ }^{10}$ The revisions to the E/M office visit code descriptors and documentation standards are now centered around how physicians think and take care of patients and not on mandatory standards and checking boxes. The main changes involve MDM as the prime determinant of the coding level. Elements of MDM affecting coding for an outpatient or office visit now include only 3 components: the number and complexity of problems addressed in the encounter, the amount or complexity of data to be reviewed and analyzed, and the risk of complications or morbidity of patient management. Gone are the requirements from the earlier criteria requiring so many bullet points for the history, physical examination, and MDM.
Dermatologists may ask, "How does the new E/M coding structure affect reporting and documenting an $\mathrm{E} / \mathrm{M}$ and a procedure on the same day?" The answer is that the determination of separate and distinct is basically unchanged with the new E/M codes; however, the documentation requirements for modifier -25 using the new E/M codes are simplified.

As always, the key to determining whether a separate and distinct E/M service was provided and subsequently documented is to deconstruct the medical note. All evaluation services associated with the procedure-making a clinical diagnosis or differential diagnosis, decision to perform surgery, and discussion of alternative treatmentsshould be removed from one's documentation as shown in the example below. If a complete E/M service still exists, then an E/M may be billed in addition to the procedure.

Physical examination of the treatment area is included in the surgical package. With the prior E/M criteria, physical examination of the procedural area could not be used again as a bullet point to count for the E/M level. However, with the new 2021 coding requirements, the documentation of a separate MDM will be sufficient to meet criteria because documentation of physical examination is not a requirement.

\section{Modifier-25 Examples}

Let's examine a typical dermatologist medical note. An established patient presents to the dermatologist complaining of an itchy rash on the left wrist after a hiking trip. Treatment with topical hydrocortisone 1\% did not help. The patient also complains of a growing tender lesion on the left elbow of 2 months' duration. Physical examination reveals a linear vesicular eruption on the left wrist and a tender hyperkeratotic papule on the left elbow. No data is evaluated. A diagnosis of acute rhus dermatitis of the left wrist is made, and betamethasone cream is prescribed. The decision is made to perform a tangential biopsy of the lesion on the left elbow because of the suspicion for malignancy. The biopsy is performed the same day.

This case clearly illustrates performance of an E/M service in the treatment of rhus dermatitis, which is separate and distinct from the biopsy procedure; however, in evaluating whether the case meets the documentation requirements for modifier -25 , the information in the medical note inclusive to the procedure's global surgical package, including history associated with establishing the diagnosis, physical examination of the procedure area(s), and discussion of treatment options, is eliminated, leaving the following notes: An established patient presents to the dermatologist complaining of an itchy rash on the left wrist after a hiking trip. Treatment with topical hydrocortisone 1\% did not help. No data is evaluated. A diagnosis of acute rhus dermatitis of the left wrist is made, and betamethasone cream is prescribed.

Because the physical examination of the body part (left arm) is included in the procedure's global surgical package, the examination of the left wrist cannot be used as coding

CONTINUED ON PAGE 162 


\section{CONTINUED FROM PAGE 132}

support for the E/M service. This makes a difference for coding level in the prior E/M coding requirements, which required examination bullet points. However, with the 2021 E/M codes, documentation of physical examination bullet points is irrelevant to the coding level. Therefore, qualifying for a modifier -25 claim is more straightforward in this case with the new code set. Because bullet points are not integral to the $2021 \mathrm{E} / \mathrm{M}$ codes, qualifying and properly documenting for a higher level of service will likely be more common in dermatology.

\section{Final Thoughts}

Frequent use of modifier -25 is a critical part of a highquality and cost-effective dermatology practice. Same-day performance of minor procedures and E/M services allows for more rapid and efficient diagnosis and treatment of various conditions as well as minimizing unnecessary office visits. The new E/M codes for 2021 actually make the documentation of a separate and distinct E/M service less complicated because the bullet point requirements associated with the old E/M codes have been eliminated. Understanding how the new E/M code descriptors affect modifier -25 reporting and clear documentation of separate, distinct, and medically necessary E/M services will be needed due to increased insurer scrutiny and audits.

\section{REFERENCES}

1. Current Procedural Terminology 2021, Professional Edition. American Medical Association; 2020.

2. Rogers HW. Modifier -25 victory, but the battle is not over. Cutis. 2018;101:409-410.
3. Rogers HW. One diagnosis and modifier -25: appropriate or audit target? Cutis. 2017;99:165-166.

4. Update regarding E/M with modifier -25-professional. Anthem Blue Cross Blue Shield website. Published February 1, 2019. Accessed August 17, 2021. https://providernews.anthem.com/ohio/article /update-regarding-em-with-modifier-25-professional

5. Payment policies-surgery. Harvard Pilgrim Health Care website. Updated May 2021. Accessed August 17, 2021. https://www .harvardpilgrim.org/provider/wp-content/uploads/sites/7/2020/07 /H-6-Surgery-PM.pdf

6. Modifier 25: frequently asked questions. Independence Blue Cross website. Updated September 25, 2017. Accessed August 17, 2021. https://provcomm.ibx.com/ibc/archive/pages/A86603B03881756B8525 817E00768006.aspx

7. Huang G. CMS 2019 fee schedule takes modifier 25 cuts, runs with them. Doctors Management website. Accessed August 17, 2021. https://www.doctors-management.com/cms-2019-feeschedule -modifier25/

8. Dermatologist claims for evaluation and management services on the same day as minor surgical procedures. US Department of Health and Humans Services Office of Inspector General website. Accessed August 17, 2021. https://oig.hhs.gov/reports-and-publications /workplan/summary/wp-summary-0000577.asp

9. Global surgery booklet. Centers for Medicare and Medicaid Services website. Updated September 2018. Accessed August 17, 2021. https://www.cms.gov/outreach-and-education/medicare-learning -network-m/n/mlnproducts/downloads/globallsurgery-icn907166.pdf

10. American Medical Association. CPT® Evaluation and management (E/M)-office or other outpatient (99202-99215) and prolonged services (99354, 99355, 99356, 99417) code and guideline changes. Updated March 9, 2021. Accessed August 17, 2021. https://www.ama-assn.org/system/files/2019-06/cpt-office-prolongedsvs-code-changes.pdf

11. 1997 documentation guidelines for evaluation and management services. Centers for Medicare and Medicaid Services website. Accessed August 17, 2021. https://www.cms.gov/Outreach-and -Education/Medicare-Learning-Network-MLN/MLNEdWebGuide /Downloads/97Docguidelines.pdf 